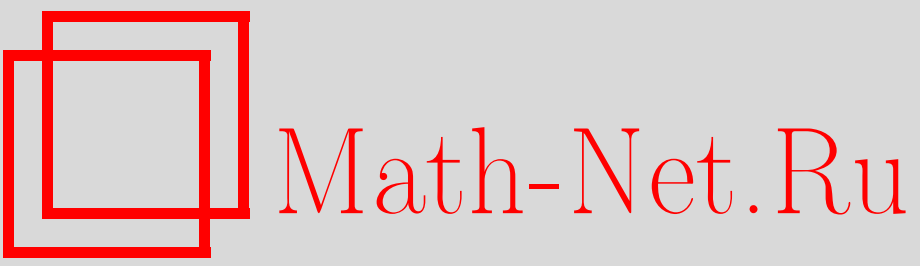

В. М. Елеонский, В. Г. Королев, Н. Е. Кулагин, О динамических системах на двумерной сфере, связанных с дробными гамильтонианами, ТМФ, 2003, том 136, номер 2, 271-284

DOI: https://doi.org/10.4213/tmf219

Использование Общероссийского математического портала Math-Net.Ru подразумевает, что вы прочитали и согласны с пользовательским соглашением

http: //www . mathnet.ru/rus/agreement

Параметры загрузки:

IP : 54.174 .149 .18

26 апреля 2023 г., 15:42:39 


\section{О ДИНАМИЧЕСКИХ СИСТЕМАХ НА ДВУМЕРНОЙ СФЕРЕ, СВЯЗАННЫХ С ДРОБНЫМИ ГАМИЛЬТОНИАНАМИ}

Рассмотрен класс "дробных" гамильтоновых систем, обобщающий классическую задачу о движении в центральном поле. Анализ основан на преобразовании интегрируемой гамильтоновой системы с двумя степенями свободы на плоскости в динамическую систему, определенную на сфере и наследующую интегралы движения исходной системы. Показано, что сушествует одномерное многообразие в четырехмерном пространстве структурных параметров (содержащее случай плоской задачи Кеплера), вдоль которого сохраняются замкнутость орбит всех финитных движений и справедливость третьего закона Кеплера. Аналогично существует одномерное многообразие (содержащее двумерный изотропный гармонический осциллятор), вдоль которого сохраняются замкнутость орбит и изохронность колебаний. Деформация орбит на выделенных многообразиях не нарушает скрытой симметрии, характерной для двумерного изотропного осциллятора и плоской задачи Кеплера. Рассмотрены также двумерные многообразия, на которых все системы характеризуются одинаковым числом вращения орбит всех финитных движений.

Ключевые слова: задача Кеплера, дробные гамильтоновы системы, изохронное движение.

\section{1. ВВЕДЕНИЕ}

Известно [1], [2], что в одномерном случае гармонический осциллятор является единственным представителем гамильтоновых систем вида $H=p^{2} / 2+U(x), U(-x)=U(x)$, который удовлетворяет условию изохронности (независимости периода колебаний $T$ от энергии). Ситуация меняется при расширении класса функций Гамильтона. Например, нетрудно показать, что для функций Гамильтона

$$
H=\frac{1}{\alpha}|p|^{\alpha}+\frac{1}{\beta}|x|^{\beta}, \quad x \in \mathbb{R}^{1},
$$

где $\alpha>0, \beta>0$ - произвольные вещественные числа, период колебаний равен

$$
T=4 \alpha^{-1+1 / \alpha} \beta^{-1+1 / \beta} \frac{\Gamma(1 / \alpha) \Gamma(1 / \beta)}{\Gamma(1 / \alpha+1 / \beta)} \mathcal{E}^{1 / \alpha+1 / \beta-1},
$$

${ }^{*}$ Научно-исследовательский институт физических проблем им. Ф. В. Лукина, Зеленоград, Москва, Россия. E-mail: korolev@nonlin.msk.ru; kulagin@nonlin.msk.ru 
где $\mathcal{E}$ - полная энергия, $\Gamma(x)$ - гамма-функция. Изохронность колебаний (уже не гармонических) реализуется на кривой $\alpha^{-1}+\beta^{-1}=1$ в плоскости структурных параметров $(\alpha, \beta)$ функций Гамильтона (1); частным случаем является гармонический осциллятор $(\alpha=\beta=2)$.

В классе гамильтоновых систем с двумя степенями свободы $H=|\vec{p}|^{2} / 2+U(|\vec{r}|)$ задача Кеплера $(U=\sigma /|\vec{r}|, \sigma<0)$ является одной из двух (наряду с изотропным гармоническим осциллятором) систем, в которых орбиты всех финитных движений замкнуты. Известно [1], [2], что это связано со скрытой симметрией (дополнительным вырождением) задачи Кеплера, обусловленной сушествованием третьего интеграла движения (помимо интегралов энергии и момента). Любые структурные возмушения в этом классе гамильтонианов приводят к возникновению прецессии орбит и исчезновению скрытой симметрии. Однако, как и в предыдушем примере, расширение класса гамильтонианов может сушественно изменить ситуацию. Ниже рассмотрен класс гамильтонианов

$$
H=p^{\alpha_{1}} r^{\beta_{1}}+\sigma p^{\alpha_{2}} r^{\beta_{2}}, \quad r=|\vec{r}|, \quad p=|\vec{p}|, \quad \vec{r}, \vec{p} \in \mathbb{R}^{2},
$$

где $\alpha_{1}, \beta_{1}, \alpha_{2}, \beta_{2}, \sigma$-вешественные параметры. Этот класс включает в себя, в частности, гамильтониан частицы в центральном поле с потенциалом $U \sim r^{\beta_{2}}, \alpha_{1}=2, \beta_{1}=$ $\alpha_{2}=0$.

Показано, что при $\sigma<0$ сушествует однопараметрическое семейство гамильтонианов вида (3), для которых траектории всех финитных орбит замкнуты и справедлив третий закон Кеплера: $T \propto(-\mathcal{E})^{-3 / 2}$. При изменении параметра семейства орбиты деформируются (в частных случах они могут иметь форму эллипсов Кеплера, окружностей или улиток Паскаля). Возможно возникновение орбит с точками самопересечения. Но при этом все представители семейства обладают скрытой симметрией (дополнительным интегралом движения).

При $\sigma>0$ сушествует однопараметрическое семейство функций Гамильтона вида (3), наследуюших от двумерного изотропного гармонического осциллятора, наряду с замкнутостью орбит, изохронность колебаний (уже нелинейных) при всех $\mathcal{E}>0$. Все представители этого семейства также обладают скрытой симметрией (дополнительным интегралом движения).

Более того, в каждом из случаев $\sigma<0$ и $\sigma>0$ соответствующее однопараметрическое семейство является частным случаем некоторого двухпараметрического семейства функций Гамильтона, для каждой из которых все финитные движения характеризуются одинаковым числом врашения. Орбиты всех финитных движений замкнуты, если это число рационально, и разомкнуты в противном случае.

Для исследования гамильтоновых систем (3) мы используем обобщение подхода, предложенного нами ранее [3], [4]. Подход основан на преобразовании интегрируемой гамильтоновой системы с двумя степенями свободы, определенной на плоскости, в динамическую систему, определенную на сфере и наследующую интегралы движения исходной системы. Динамическая система на сфере не только обладает рядом преимуществ с точки зрения ее качественного и численного анализа, но и представляет самостоятельный 
интерес как пример динамической системы с полутора степенями свободы, обладающей двумя первыми интегралами. Более того, первые интегралы этой системы на сфере позволяют выявить законы подобия по отношению к одной из угловых переменных, связанные с линейными преобразованиями одномерных и двумерных многообразий в себя в пространстве структурных параметров $\left(\alpha_{1}^{0}, \beta_{1}^{0}, \alpha_{2}^{0}, \beta_{2}^{0}\right)$. Результаты численных расчетов траекторий динамической системы на сфере находятся в согласии с рассчитанными траекториями исходной гамильтоновой системы.

\section{2. ДИНАМИЧЕСКАЯ СИСТЕМА НА СФЕРЕ}

Канонические уравнения для гамильтонианов вида $H=H(p, r)$ имеют вид

$$
\dot{\vec{r}}=\frac{\partial H}{\partial p} \frac{\vec{p}}{p}, \quad \dot{\vec{p}}=-\frac{\partial H}{\partial r} \frac{\vec{r}}{r}
$$

и обладают двумя интегралами движения (энергией $\mathcal{E}=H$ и моментом $\vec{M}=[\vec{r}, \vec{p}]$ ). Определив векторы $\vec{p}$ и $\vec{r}$ в полярных координатах $(p, \psi)$ и $(r, \varphi)$, приходим к неканоническим уравнениям движения

$$
\begin{aligned}
\dot{r} & =\frac{\partial H}{\partial p} \cos (\psi-\varphi), & \dot{p} & =-\frac{\partial H}{\partial r} \cos (\psi-\varphi), \\
r \dot{\varphi} & =\frac{\partial H}{\partial p} \sin (\psi-\varphi), & p \dot{\psi} & =\frac{\partial H}{\partial r} \sin (\psi-\varphi) .
\end{aligned}
$$

Переход к новым угловым переменным $\Psi=\psi+\varphi, \Phi=\psi-\varphi$ приводит к следуюшей записи уравнений движения:

$$
\begin{aligned}
\dot{r} & =\frac{\partial H}{\partial p} \cos \Phi, & \dot{p} & =-\frac{\partial H}{\partial r} \cos \Phi, \\
\dot{\Phi} & =\left(\frac{1}{p} \frac{\partial H}{\partial r}-\frac{1}{r} \frac{\partial H}{\partial p}\right) \sin \Phi, & \dot{\Psi} & =\left(\frac{1}{p} \frac{\partial H}{\partial r}+\frac{1}{r} \frac{\partial H}{\partial p}\right) \sin \Phi,
\end{aligned}
$$

которая указывает на возможность редукции к динамической системе с полутора степенями свободы.

Если используется гамильтониан вида (3), исключение $p$ и $r$ в уравнениях движения (6) приводит к следуюшей системе уравнений для угловых переменных $(\Psi, \Phi)$ :

$$
\begin{aligned}
& \ddot{\Phi}=\operatorname{ctg} \Phi\left\{2(\dot{\Phi})^{2}+\frac{\theta_{1}-\theta_{2}}{4 D}\left(\zeta_{1} \dot{\Phi}-\theta_{1} \dot{\Psi}\right)\left(\zeta_{2} \dot{\Phi}-\theta_{2} \dot{\Psi}\right)\right\} \\
& \ddot{\Psi}=\operatorname{ctg} \Phi\left\{2 \dot{\Phi} \dot{\Psi}+\frac{\zeta_{1}-\zeta_{2}}{4 D}\left(\zeta_{1} \dot{\Phi}-\theta_{1} \dot{\Psi}\right)\left(\zeta_{2} \dot{\Phi}-\theta_{2} \dot{\Psi}\right)\right\} .
\end{aligned}
$$

Здесь

$$
\zeta_{i}=\beta_{i}+\alpha_{i}, \quad \theta_{i}=\beta_{i}-\alpha_{i}, \quad i=1,2, \quad D=\beta_{2} \alpha_{1}-\beta_{1} \alpha_{2} \neq 0 .
$$

4 Теоретическая и математическая физика, т. 136, № 2, 2003 г. 
Конфигурационное пространство динамической системы (7) отождествим с поверхностью единичной сферы ( $\Phi$ и $\Psi$ - полярный и азимутальный углы). Состояние системы определяется положением точки на сфере и вектором угловой скорости в плоскости, касательной к сфере в точке $(\Phi, \Psi)$.

Подчеркнем, что произвольные действительные показатели степени $\alpha_{i}, \beta_{i}$ в функции Гамильтона (3) входят только в коэффициенты уравнений (7), что сушественно облегчает анализ последних.

Значения модулей векторов $\vec{r}$ и $\vec{p}$ связаны с угловыми переменными $(\Phi, \Psi)$ соотношениями

$$
\begin{aligned}
& r^{D-\left(\theta_{2}-\theta_{1}\right)}=\left(\frac{\theta_{2} \dot{\Psi}-\zeta_{2} \dot{\Phi}}{2 D \sin \Phi}\right)^{1-\alpha_{2}}\left(\frac{\theta_{1} \dot{\Psi}-\zeta_{1} \dot{\Phi}}{-2 \sigma D \sin \Phi}\right)^{\alpha_{1}-1} \\
& p^{D-\left(\theta_{2}-\theta_{1}\right)}=\left(\frac{\theta_{2} \dot{\Psi}-\zeta_{2} \dot{\Phi}}{2 D \sin \Phi}\right)^{\beta_{2}-1}\left(\frac{\theta_{1} \dot{\Psi}-\zeta_{1} \dot{\Phi}}{-2 \sigma D \sin \Phi}\right)^{1-\beta_{1}} .
\end{aligned}
$$

Условия $r>0, p>0$ выделяют на плоскости угловых скоростей $(\dot{\Psi}, \dot{\Phi})$ допустимые области их значений:

$$
D\left(\theta_{2} \dot{\Psi}-\zeta_{2} \dot{\Phi}\right)>0, \quad D \sigma\left(\theta_{1} \dot{\Psi}-\zeta_{1} \dot{\Phi}\right)>0
$$

Можно показать, что динамическая система (7) обладает двумя интегралами движения

$$
\begin{aligned}
& I_{1}=\frac{1}{D \sin ^{2} \Phi}\left[\left(\theta_{2}-\theta_{1}\right) \dot{\Psi}-\left(\zeta_{2}-\zeta_{1}\right) \dot{\Phi}\right] \\
& I_{2}=(\sin \Phi)^{D-2\left(\theta_{2}-\theta_{1}\right)}\left(\frac{\theta_{2} \dot{\Psi}-\zeta_{2} \dot{\Phi}}{2 D}\right)^{\theta_{2}}\left(\frac{\theta_{1} \dot{\Psi}-\zeta_{1} \dot{\Phi}}{-2 \sigma D}\right)^{-\theta_{1}} .
\end{aligned}
$$

При этом

$$
I_{1}=\frac{2 \mathcal{E}}{M}, \quad I_{2}=M^{D-\left(\theta_{2}-\theta_{1}\right)}
$$

Здесь и далее предполагается, что $M \neq 0$ и учтены условия (10), исключаюшие отрицательные значения оснований всех степеней. Кроме того, мы будем предполагать, что $\theta_{1} \neq \theta_{2}$. (Условие $\theta_{1}=\theta_{2}$ выделяет в четырехмерном пространстве структурных параметров трехмерное многообразие, требующее отдельного анализа. В частности, этому многообразию отвечают системы, в которых реализуется падение на центр (пример: $\left.H=p^{2}-1 / r^{2}\right)$.)

Исключая из первого уравнения в (11) угловую скорость $\dot{\Psi}$, приходим к следуюшей записи уравнений (11):

$$
\begin{aligned}
1 & =\left(\frac{\sin \Phi}{\sin \Phi_{0}}\right)^{D}\left(1-\frac{1}{\nu_{2}} \frac{\Phi^{\prime}}{\sin ^{2} \Phi}\right)^{\nu_{2} D}\left(1-\frac{1}{\nu_{1}} \frac{\Phi^{\prime}}{\sin ^{2} \Phi}\right)^{-\nu_{1} D}, \\
\Psi^{\prime} & =\frac{2}{D} \frac{\sin ^{2} \Phi}{\nu_{2}-\nu_{1}}+\frac{\zeta_{2}-\zeta_{1}}{\nu_{2}-\nu_{1}} \Phi^{\prime},
\end{aligned}
$$

где

$$
\Phi^{\prime}=\frac{d \Phi}{d \tau}, \quad \tau=\frac{M}{\mathcal{E} D} t, \quad \nu_{1}=\frac{\theta_{1}}{D}, \quad \nu_{2}=\frac{\theta_{2}}{D}
$$


а величина $\sin ^{2} \Phi_{0}<1$ определена (для финитных движений) соотношением

$$
1=\frac{\mathcal{E}^{\left(\nu_{2}-\nu_{1}\right) D}}{M^{D}}\left(\sin ^{2} \Phi_{0}\right)^{D}\left(\frac{\nu_{2}}{\nu_{2}-\nu_{1}}\right)^{\nu_{2} D}\left(\frac{\nu_{1}}{(-\sigma)\left(\nu_{2}-\nu_{1}\right)}\right)^{-\nu_{1} D}
$$

Учитывая, что уравнение (13) и соотношение (16) допускают формальную операцию извлечения корня степени $D$, мы получаем, что

$$
\begin{aligned}
& \Phi(\tau)=\Phi\left(\tau ; \nu_{1}, \nu_{2} \mid \mathcal{E}, M\right) \\
& \Psi(\tau)=\Psi\left(\tau ; \nu_{1}, \nu_{2}, D, \zeta_{2}-\zeta_{1} \mid \mathcal{E}, M\right) .
\end{aligned}
$$

Обшее движение изображающей точки на сфере представляет собой врашение по азимутальному углу $\Psi$, сопровождаемое периодическими (с периодом $\bar{T}$ ) колебаниями по полярному углу $\Phi: \Phi(\tau+\bar{T})=\Phi(\tau)$, где $\bar{T}=\bar{T}\left(\nu_{1}, \nu_{2}, \sin \Phi_{0}\right) ;$ при этом в общем случае $\Psi(\tau+\bar{T}) \neq \Psi(\tau)$. Уравнение (13) может быть разрешено относительно величины $\sin \Phi$, которую можно рассматривать как функцию от $\Phi^{\prime}$. Тогда, делая замену $\Phi \rightarrow z=\operatorname{ctg} \Phi$, мы получаем выражение для периода колебаний $T(T=\bar{T} \mathcal{E} /(M D))$ полярного угла $\Phi(t)$ :

$$
T=\frac{1 / \nu_{1}-1 / \nu_{2}}{\sin \Phi_{0}} \int_{z_{-}}^{z_{+}} d z \frac{\left(1-z / \nu_{2}\right)^{2 \nu_{2}-1}\left(1-z / \nu_{1}\right)^{-2 \nu_{1}-1}}{\sqrt{\left(1-z / \nu_{2}\right)^{2 \nu_{2}}\left(1-z / \nu_{1}\right)^{-2 \nu_{1}-\sin ^{2} \Phi_{0}}}}
$$

Определяя число вращения $N$ траектории на сфере как приращение азимутального угла $\Psi$ на периоде полярного угла $\Phi$, отнесенное к $2 \pi$, находим

$$
N=\frac{\sin \Phi_{0}}{\pi \nu_{1} \nu_{2} D} \int_{z_{-}}^{z_{+}} d z \frac{\left(1-z / \nu_{2}\right)^{-1}\left(1-z / \nu_{1}\right)^{-1}}{\sqrt{\left(1-z / \nu_{2}\right)^{2 \nu_{2}}\left(1-z / \nu_{1}\right)^{-2 \nu_{1}-\sin ^{2} \Phi_{0}}}} .
$$

В этих выражениях $z_{ \pm}-$корни уравнения

$$
\left(1-\frac{z}{\nu_{2}}\right)^{2 \nu_{2}}\left(1-\frac{z}{\nu_{1}}\right)^{-2 \nu_{1}}=\sin ^{2} \Phi_{0}
$$

Подчеркнем, что, несмотря на то что функция $\Psi(t)$ зависит от комбинации $\zeta_{2}-\zeta_{1}$, число врашения $N$ от нее не зависит. Это означает, что, зафиксировав в четырехмерном пространстве структурных параметров $\left(\alpha_{1}, \beta_{1}, \alpha_{2}, \beta_{2}\right)$ значения трех их комбинаций

$$
\theta_{1}=\beta_{1}-\alpha_{1}, \quad \theta_{2}=\beta_{2}-\alpha_{2}, \quad D=\alpha_{1} \beta_{2}-\alpha_{2} \beta_{1}
$$

мы получаем одномерные многообразия, вдоль которых сохраняется зависимость числа врашения $N$ от постоянных первых интегралов $\mathcal{E}$ и $M$ (см. следующий раздел). 


\section{3. ПРИМЕРЫ: ДРОБНЫЕ ПРОДОЛЖЕНИЯ ЗАДАЧИ КЕПЛЕРА И ГАРМОНИЧЕСКОГО ОСЦИЛЛЯТОРА}

3.1. Одномерные многообразия. В силу выражений (13), (15), (17) уравнения для полярного угла $\Phi$ зависят только от приведенных в (21) трех комбинаций структурных параметров $\left(\alpha_{1}^{0}, \beta_{1}^{0}, \alpha_{2}^{0}, \beta_{2}^{0}\right)$. Они постоянны на прямых

$$
\alpha_{1}=\alpha_{1}^{0}+k_{1} \gamma, \quad \beta_{1}=\beta_{1}^{0}+k_{1} \gamma, \quad \alpha_{2}=\alpha_{2}^{0}+k_{2} \gamma, \quad \beta_{2}=\beta_{2}^{0}+k_{2} \gamma
$$

в четырехмерном пространстве структурных параметров ( $\gamma$-параметр прямой, $k_{2} / k_{1}=$ $\left.\left(\beta_{2}-\alpha_{2}\right) /\left(\beta_{1}-\alpha_{1}\right) \equiv k_{0}\right)$. На каждой такой прямой сохраняется зависимость периода колебаний угла $\Phi$ (а в силу $(19),(20)$ и числа врашения $N$ ) от постоянных первых интегралов $\mathcal{E}, M$. Прямым (22) отвечают функции Гамильтона

$$
H=p^{\alpha_{1}^{0}} r^{\beta_{1}^{0}}(r p)^{k_{1} \gamma}+\sigma p^{\alpha_{2}^{0}} r_{2}^{\beta_{2}^{0}}(r p)^{k_{2} \gamma} .
$$

Рассмотрим в качестве примеров семейства динамических систем, отвечающих двум прямым (22), проходяшим через точки, соответствуюшие плоской задаче Кеплера и задаче об изотропном осцилляторе.

3.2. Дробное продолжение задачи Кеплера. На прямой (22), проходящей через точку $\left(\alpha_{1}^{0}=2, \beta_{1}^{0}=\alpha_{2}^{0}=0, \beta_{2}^{0}=-1\right)$, отвечаюшей задаче Кеплера, имеем $D=-2$, $\nu_{1}=+1, \nu_{2}=1 / 2$; при этом

$$
\alpha_{1}=2+\gamma, \quad \beta_{1}=\gamma, \quad \alpha_{2}=\frac{\gamma}{2}, \quad \beta_{2}=-1+\frac{\gamma}{2} .
$$

На этой прямой уравнение (13) разрешимо относительно $\Phi^{\prime}$ :

$$
\frac{M}{2 \mathcal{E}} \frac{\Phi^{\prime}}{\sin ^{2} \Phi}=\frac{\sigma^{2}}{2 M^{3}} \sqrt{\sin ^{2} \Phi-\sin ^{2} \Phi_{0}}\left[\sqrt{\sin ^{2} \Phi-\sin ^{2} \Phi_{0}} \pm \sin \Phi\right],
$$

где $\sin ^{2} \Phi_{0}=-4 \mathcal{E} M^{2} / \sigma^{2}$. Интегрируя это уравнение, определяем две ветви функции $t=t(\Phi)$ :

$$
\frac{\sigma^{2}\left(\sin \Phi_{0}\right)^{3}}{2 M^{3}}\left(t-t_{i}\right)=\left.\left\{\sin \Phi_{0} \operatorname{ctg} \Phi \mp \operatorname{arctg} \frac{\operatorname{ctg} \Phi}{\sqrt{\operatorname{ctg}^{2} \Phi_{0}-\operatorname{ctg}^{2} \Phi}}\right\}\right|_{\Phi_{i}} ^{\Phi} .
$$

Интегрируя по циклу $\Phi_{0}^{-} \Rightarrow \Phi_{0}^{+} \Rightarrow \Phi_{0}^{-}\left(0<\Phi_{0}^{-} \leqslant \pi / 2 \leqslant \Phi_{0}^{+}<\pi\right)$, находим, что период колебаний $\Phi(t)$ подчиняется зависимости $T \propto|\sigma|(-\mathcal{E})^{-3 / 2}$, т.е. третьему закону Кеплера, при любом $\gamma$. Рассматривая $\Psi$ как функцию $\Phi$, находим, что две ее ветви определяются уравнениями

$$
\frac{d \Psi}{d \Phi}+\gamma-1= \pm \frac{2 \sin \Phi}{\sqrt{\sin ^{2} \Phi-\sin ^{2} \Phi_{0}}} .
$$

Интегрируя по циклу $\Phi_{0}^{-} \Rightarrow \Phi_{0}^{+} \Rightarrow \Phi_{0}^{-}$, находим, что прирашение азимутального угла $\Psi$ на периоде $T$ полярного угла равно $2 \pi$ независимо от $\mathcal{E}, M$ и параметра $\gamma$. Таким 
образом, в случае (24) все финитные орбиты на сфере замкнуты при любом $\gamma$. Однако форма орбит на сфере зависит от $\gamma$ :

$$
\cos \Phi= \pm\left|\cos \Phi_{0}\right| \sin \left[\frac{1}{2} \Psi+\frac{1}{2}(1+\gamma) \Phi+\text { const }\right] .
$$

Далее, можно показать, что

$$
r(\Phi, \gamma)=\left(\frac{M}{\sin \Phi}\right)^{1+\gamma / 2} r^{0}(\Phi)
$$

где $r^{0}(\Phi)$ определено соотношением

$$
\frac{1}{r^{0}(\Phi)}=\frac{|\sigma|}{2 M}\left[\sin \Phi \pm \sqrt{\sin ^{2} \Phi-\sin ^{2} \Phi_{0}}\right] .
$$

Эволюция полярного угла $\varphi$ в исходной полярной системе координат на плоскости $(r, \varphi)$ определяется выражением $\varphi=(\Psi(\sin \Phi, \gamma)-\Phi) / 2$, в котором $\Psi(\sin \Phi, \gamma)$ - решение уравнения (27). Отсюда следует, что условия замыкания орбиты на плоскости $(r, \varphi)$

$$
r(t+2 T, \gamma)=r(t, \gamma), \quad \varphi(t+2 T, \gamma)=\varphi(t, \gamma)
$$

выполняются при всех значениях $\gamma$.

Наконец, приведем формулы преобразования орбит на плоскости $(r, \varphi)$ при изменении параметра $\gamma$ :

$$
\begin{aligned}
r & =2\left(\frac{M}{\sin \Phi}\right)^{\gamma} r^{0}(\Phi), \quad r^{\prime}=2\left(\frac{M}{\sin \Phi}\right)^{\gamma^{\prime}} r^{0}(\Phi), \\
\Phi & =\Phi_{0}^{-}-2 \frac{\varphi^{\prime}-\varphi}{\gamma^{\prime}-\gamma}
\end{aligned}
$$

где $(r, \varphi)$ и $\left(r^{\prime}, \varphi^{\prime}\right)$ - точки соответствующих орбит для динамических систем с гамильтонианами

$$
H=p^{2}(r p)^{\gamma}+\frac{\sigma}{r}(r p)^{\gamma / 2}, \quad H=p^{2}(r p)^{\gamma^{\prime}}+\frac{\sigma}{r}(r p)^{\gamma^{\prime} / 2} .
$$

Эти формулы позволяют найти выражение для третьего интеграла движения при $\gamma \neq 0$ по известному выражению для случая $\gamma=0$ (т.е. задачи Кеплера). Это означает, что на прямой (24) все динамические системы сохраняют скрытую симметрию задачи Кеплера.

На рисунке изображена деформация орбит на плоскости $(r, \varphi)$ при изменении параметра $\gamma$, подтвержденная численными расчетами: (а) $\gamma=0$ (задача Кеплера); (б) $\gamma=$ 0.3 ; (в) $\gamma=0.9$; (г) $\gamma=2$; (д) $\gamma=4$; (е) $\gamma=-0.9$; (ж) $\gamma=-2$ (все орбиты - окружности с центром, смешенным относительно начала координат); (з) $\gamma=-4$ (все орбиты имеют форму улиток Паскаля); (и) $\gamma=-8$. Возникновение особенностей на орбитах связано с реализацией в определенных интервалах значений $\gamma$ условий $\dot{r}=0, \dot{\varphi}=0$ (т.е. $\Phi=\pi / 2$, $\partial H / \partial p=0)$. Отметим, что "возвратное" движение по орбите (смена знака $\dot{\varphi})$ не связано 


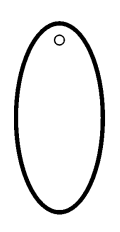

a

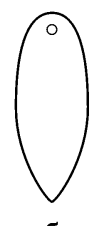

б

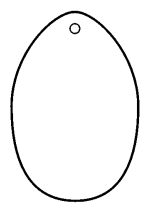

e

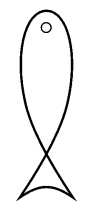

B

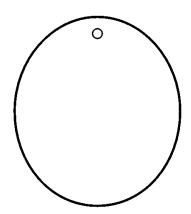

अ

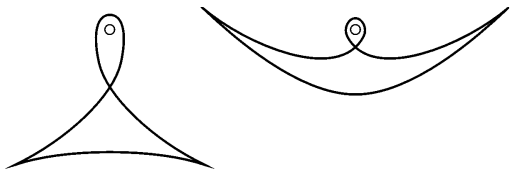

$\Gamma$

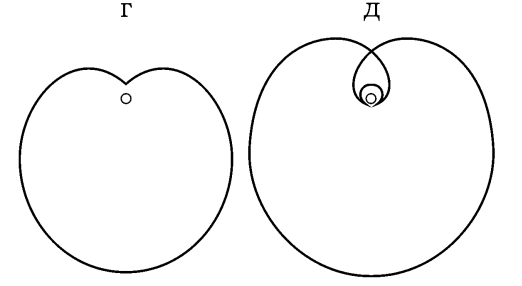

3

И

с изменением направления вектора момента импульса: на участках “обратного" движения вектор скорости направлен противоположно вектору импульса.

3.3. Дробное продолжение задачи о гармоническом осцилляторе. Рассмотрим теперь прямую (22), проходящую через точку $\left(\alpha_{1}^{0}=2, \beta_{1}^{0}=\alpha_{2}^{0}=0, \beta_{2}^{0}=2\right)$, которая отвечает задаче о гармоническом осцилляторе. На этой прямой $D=4, \nu_{1}=$ $-1 / 2, \nu_{2}=1 / 2 ;$ при этом

$$
\alpha_{1}=2+\gamma, \quad \beta_{1}=\gamma, \quad \alpha_{2}=-\gamma, \quad \beta_{2}=2-\gamma
$$

В этом случае уравнения (13), (14) принимают вид

$$
\dot{\Phi}= \pm 2 \sigma^{1 / 2} \sin \Phi \sqrt{\frac{\sin ^{2} \Phi}{\sin ^{2} \Phi_{0}} \pm 1}, \quad \dot{\Psi}=\frac{2 \mathcal{E}}{M} \sin ^{2} \Phi-\gamma \dot{\Phi}
$$

Здесь $\sin ^{2} \Phi_{0}=\sigma M^{2} / \mathcal{E}^{2}$, а дифференцирование проводится по исходной независимой переменной $t$. Интегрируя первое из уравнений (при выборе нижнего знака под радикалом), определяем две ветви функции $t=t(\Phi)$ :

$$
2 \sigma^{1 / 2}\left(t-t_{i}\right)=\left.\mp \operatorname{arctg} \frac{\operatorname{ctg} \Phi}{\sqrt{\operatorname{ctg}^{2} \Phi_{0}-\operatorname{ctg}^{2} \Phi}}\right|_{\Phi_{i}} ^{\Phi} .
$$

Интегрируя, как и в предыдушем примере, по циклу $\Phi_{0}^{-} \Rightarrow \Phi_{0}^{+} \Rightarrow \Phi_{0}^{-}$, находим, что период колебаний $\Phi(t)$ равен $T=\sigma^{-1 / 2}$ и не зависит от постоянных первых интегралов $\mathcal{E}, M$ и параметра $\gamma$. Азимутальный угол $\Psi$, рассматриваемый как функция $\Phi$, определяется выражением

$$
\Psi-\Psi_{i}=\left.\mp \arcsin \frac{\cos \Phi}{\left|\cos \Phi_{0}\right|}\right|_{\Phi_{i}} ^{\Phi}-\gamma\left(\Phi-\Phi_{i}\right)
$$


Приращение $\Psi$ на периоде $T$ полярного угла $\Phi$ равно $\pi$ при всех $\gamma$ (дефоормация орбит не нарушает их замкнутости). Значения $r$ и $\varphi$ восстанавливаются по формулам

$$
\begin{gathered}
r^{2}(t, \gamma)=\frac{\mathcal{E}}{2 \sigma}\left(\frac{M}{\sin \Phi}\right)^{\gamma}\left(1 \pm \sqrt{1-\frac{\sin ^{2} \Phi_{0}}{\sin ^{2} \Phi}}\right), \\
2 \varphi=\mp \arcsin \frac{\cos \Phi}{\left|\cos \Phi_{0}\right|}-(1+\gamma)\left(\Phi-\Phi_{0}^{-}\right) .
\end{gathered}
$$

При этом $r(t+T, \gamma)=r(t, \gamma)$ и $\varphi(t+T, \gamma)=\varphi(t, \gamma)+\pi$ при всех $\gamma$. Итак, и в этом случае все орбиты финитных движений замкнуты; при этом все соответствуюшие динамические системы с гамильтонианами $H=p^{2}(r p)^{\gamma}+\sigma r^{2}(r p)^{-2 \gamma}$ изохронны.

3.4. Двухпараметрическое семейство систем, отвечающее соизмеримости $\nu_{1} / \nu_{2}=2$. Рассмотрим множество прямых (22), удовлетворяющих условиям $\theta_{1}=$ $D, \theta_{2}=D / 2$, или, в явной форме, условиям

$$
\beta_{1}-\alpha_{1}=\beta_{2} \alpha_{1}-\beta_{1} \alpha_{2}, \quad \beta_{2}-\alpha_{2}=\frac{1}{2}\left(\beta_{2} \alpha_{1}-\beta_{1} \alpha_{2}\right) .
$$

Этому множеству принадлежат, в частности, точки $\left(\alpha_{1}=2, \beta_{1}=\alpha_{2}=0, \beta_{2}=-1\right)$ и $\left(\alpha_{1}=\beta_{2}=0, \beta_{1}=2, \alpha_{2}=-1\right)$, соответствуюшие задаче Кеплера в $r$ - и $p$-представлениях.

Уравнения прямых, принадлежаших этому множеству, определены в (22), где $\left(\alpha_{1}^{0}, \beta_{1}^{0}\right.$, $\left.\alpha_{2}^{0}, \beta_{2}^{0}\right)$ удовлетворяют условиям (38), а $k_{2} / k_{1}=1 / 2$. Разрешая уравнения (22) относительно, например, $\alpha_{1}^{0}$ и $\beta_{2}^{0}$, находим, что

$$
\alpha_{2}^{0}=-1+\frac{1}{2} \alpha_{1}^{0}, \quad \beta_{1}^{0}=2\left(1+\beta_{2}^{0}\right), \quad D^{0}=2\left(1+\beta_{2}^{0}\right)-\alpha_{1}^{0} \neq 0
$$

либо

$$
\alpha_{2}^{0}=\beta_{2}^{0}, \quad \beta_{1}^{0}=\alpha_{1}^{0}, \quad D^{0}=0 .
$$

Полагая $k_{1}=1, k_{2}=1 / 2$ и $D^{0} \neq 0$, находим, что искомое множество прямых определено выражениями

$$
\begin{aligned}
\alpha_{1} & =\alpha_{1}^{0}+\gamma, & \beta_{1} & =2\left(1+\beta_{2}^{0}\right)+\gamma, \\
\alpha_{2} & =-1+\frac{1}{2} \alpha_{1}^{0}+\frac{1}{2} \gamma, & \beta_{2} & =\beta_{2}^{0}+\frac{1}{2} \gamma
\end{aligned}
$$

или

$$
\begin{aligned}
\alpha_{1}-2 \alpha_{2} & =2, & \beta_{1}-2 \beta_{2} & =2, \\
\alpha_{1}-\alpha_{1}^{0} & =2\left(\beta_{2}-\beta_{2}^{0}\right), & \beta_{1}-\beta_{1}^{0} & =2\left(\alpha_{2}-\alpha_{2}^{0}\right) .
\end{aligned}
$$

Из этих выражений следует, что все прямые рассматриваемого множества принадлежат двумерному многообразию, связанному с условием $\nu_{1} / \nu_{2}=2$, а выражения (41) определяют расположение прямой, проходящей через точку $\left(\alpha_{1}^{0}, \beta_{1}^{0}, \alpha_{2}^{0}, \beta_{2}^{0}\right)$ в пространстве структурных параметров $\left(\alpha_{1}, \beta_{1}, \alpha_{2}, \beta_{2}\right)$. 
Обратим внимание на то, что при $\nu_{1} / \nu_{2}=2$ согласно выражениям (18), (19) $T \propto$ $(-\mathcal{E})^{-3 / 2} 2 D^{-1}$, а $N=2 / D$ на рассмотренном выше многообразии.

Уравнение, определяющее орбиту на сфере, принимает вид

$$
\frac{d \Psi}{d \Phi}+\Gamma= \pm \frac{D}{2} \frac{2 \sin \Phi}{\sqrt{\sin ^{2} \Phi-\sin ^{2} \Phi_{0}}}
$$

где

$$
\Gamma=\frac{2}{D}\left(2+\beta_{2}^{0}+\alpha_{2}^{0}-\beta_{1}^{0}-\alpha_{1}^{0}-\gamma\right)
$$

Сравнение этого уравнения с уравнением (27) показывает, что замыкание орбит всех финитных движений реализуется при рациональных значениях $D$.

Таким образом, любой точке с произвольными значениями $D \neq 0$ и $\gamma$ на двумерном многообразии (42) отвечает система с функцией Гамильтона

$$
H=p^{-D}(r p)^{\gamma}-|\sigma| p^{-D / 2}(r p)^{\gamma / 2-1},
$$

все финитные орбиты которой характеризуются одинаковым числом врашения $N=$ $2 /|D|$. Зафиксировав в выражениях (42) значения параметров, обеспечивающие выполнение условия $D=D^{0}$, мы получаем прямую вида (22) с параметром $\gamma$, лежашую на указанном двумерном многообразии, на которой все системы характеризуются одним и тем же числом врашения $N=2 /\left|D^{0}\right|$ (обшим для всех финитных орбит). На прямых, отвечающих рациональным значениям $D$, все финитные орбиты замкнуты, а на прямых, отвечающих иррациональным значениям $D$, все финитные орбиты разомкнуты.

В частности, на указанном двумерном многообразии лежит рассмотренная в п. 3.2 прямая (24), отвечаюшая $D=-2$ и проходяшая через точку $\left(\alpha_{1}^{0}=2, \beta_{1}^{0}=\alpha_{2}^{0}=0, \beta_{2}^{0}=\right.$ $-1)$, а также прямая, отвечающая $D=+2$ и проходящая через точку $\left(\alpha_{1}^{0}=0, \beta_{1}^{0}=2\right.$, $\left.\alpha_{2}^{0}=-1, \beta_{2}^{0}=0\right)$, соответствуюшую задаче Кеплера в импульсном представлении.

\section{4. АСИМПТОТИЧЕСКИЙ АНАЛИЗ ОРБИТ, БЛИЗКИХ К КРУГОВЫМ}

4.1. Круговые орбиты. Для любой финитной орбиты угловая переменная $\Phi$ изменяется в пределах полосы $0 \leqslant \pi / 2-\Delta \Phi \leqslant \Phi \leqslant \pi / 2+\Delta \Phi \leqslant \pi$, ширина которой определяется начальными условиями и которая, в частности, может стягиваться в экваториальную окружность. Действительно, уравнения (7) допускают решения

$$
\Phi=\Phi_{0} \equiv \frac{\pi}{2}, \quad \Psi=\Psi_{0}+\Omega_{0} t
$$

описывающие равномерное врашение изображающей точки вдоль экватора сферы с угловой скоростью $\Omega_{0}$; такие решения отвечают круговым орбитам на плоскости $(r, \varphi)$, для которых $r=r_{0}>0$ и $p=p_{0}>0$. В общем случае $\left(\theta_{1} \neq \theta_{2}\right)$

$$
r_{0}=\left(\frac{\mathcal{E} \theta_{2}}{\Delta \theta}\right)^{-\alpha_{2} / D}\left(\frac{-\mathcal{E} \theta_{1}}{\sigma \Delta \theta}\right)^{\alpha_{1} / D}, \quad p_{0}=\left(\frac{\mathcal{E} \theta_{2}}{\Delta \theta}\right)^{\beta_{2} / D}\left(\frac{-\mathcal{E} \theta_{1}}{\sigma \Delta \theta}\right)^{-\beta_{1} / D}
$$


где $\Delta \theta \equiv \theta_{2}-\theta_{1}$. Отметим, что на круговых орбитах первые интегралы $\mathcal{E}, M$ зависимы:

$$
M^{D}=\left|\frac{\mathcal{E}}{\Delta \theta}\right|^{\Delta \theta}\left|\sigma^{-1} \theta_{1}\right|^{-\theta_{1}}\left|\theta_{2}\right|^{\theta_{2}} .
$$

В вырожденном случае $\theta_{1}=\theta_{2}$ круговым орбитам отвечают уровни $\mathcal{E}=0$ и $M=$ $(-\sigma)^{\theta_{1} / D}$, которым отвечает семейство круговых орбит таких, что $r_{0} p_{0}=M$.

4.2. Асимптотический анализ. Уравнения (7) допускают построение степенных асимптотических решений в окрестности круговых орбит. Такое решение можно описать как врашение (т.е. монотонное увеличение азимутального угла $\Psi$ ) вблизи экватора сферы, сопровождаюшееся колебаниями по полярному углу $\Phi$ с малой амплитудой.

Будем искать разложения для функций $\Phi(t)$ и $\Upsilon(t) \equiv \dot{\Psi}$ в виде

$$
\Phi=\frac{\pi}{2}+\sum_{n=1}^{\infty} \varepsilon^{n} \Phi_{n}(\tau), \quad \Upsilon=C(\varepsilon)+\sum_{n=1}^{\infty} \varepsilon^{n} \Upsilon_{n}(\tau) .
$$

Константа $C$ также разлагается в ряд по степеням $\varepsilon$ :

$$
C(\varepsilon)=C_{0}+\sum_{n=1}^{\infty} \varepsilon^{n} C_{n} .
$$

Уравнение для $\Phi_{1}(\tau)$, возникающее в первом порядке по $\varepsilon$, имеет вид

$$
\frac{d^{2}}{d \tau^{2}} \Phi_{1}(\tau)+\frac{1}{4} \frac{C_{0}^{2}\left(\theta_{1}-\theta_{2}\right) \theta_{1} \theta_{2}}{D} \Phi_{1}(\tau)=0 .
$$

Налагая на искомую функцию условие $2 \pi$-периодичности, находим, что

$$
C_{0}=2 \sqrt{\frac{D}{\left(\theta_{1}-\theta_{2}\right) \theta_{1} \theta_{2}}} .
$$

Тогда $\Phi_{1}(\tau)=\sin \tau$. Аналогично, решая уравнение для функции $\Upsilon_{1}(\tau)$, получаем

$$
\Upsilon_{1}(\tau)=\frac{\zeta_{1}-\zeta_{2}}{\theta_{1}-\theta_{2}} \cos \tau
$$

Исключая секулярное слагаемое в уравнении для $\Phi_{2}$, находим, что $C_{1}=0$ и

$$
\Phi_{2}(\tau)=\frac{1}{6} \sqrt{\frac{D}{\left(\theta_{1}-\theta_{2}\right) \theta_{1} \theta_{2}}}\left(\theta_{1}+\theta_{2}\right) \sin 2 \tau .
$$

Аналогично определяется $\Upsilon_{2}$. Исключая секулярное слагаемое в уравнении для $\Phi_{3}(\tau)$, получаем

$$
C_{2}=\frac{1}{12} \sqrt{\frac{D}{\left[\left(\theta_{1}-\theta_{2}\right) \theta_{1} \theta_{2}\right]^{3}}}\left[D\left(\theta_{1}^{2}-\theta_{1} \theta_{2}+\theta_{2}^{2}\right)-3 \theta_{1} \theta_{2}\left(\theta_{1}-\theta_{2}\right)\right] .
$$


Для орбит, близких к круговой, рассмотрим зависимость их чисел вращения $N$ от малого параметра $\varepsilon$. На плоскости $(r, \varphi)$ число врашения определяют как угловое расстояние $\Delta \varphi$ между двумя последовательными апоцентрами (точками, где $r=r_{\max }$ ), отнесенное к $2 \pi$. В силу уравнений движения точкам апоцентра отвечают на сфере точки пересечения траекторией экватора при переходе из "южного" полушария (с полюсом $\Phi=0$ ) в "северное" (с полюсом $\Phi=\pi$ ). Таким образом, число вращения дается формулой

$$
N=\frac{1}{4 \pi}\left\{\left.\Psi(\tau)\right|_{\tau=2 \pi}-\left.\Psi(\tau)\right|_{\tau=0}\right\}
$$

Учитывая полученные асимптотические разложения, находим, что для орбит, близких к круговой, $N=N(\varepsilon) \approx C(\varepsilon) / 2$. Для само́й круговой орбиты число врашения не определено. Доопределим его как предел

$$
N_{0}=\lim _{\varepsilon \rightarrow 0} N(\varepsilon)=\frac{1}{2} C_{0}=\sqrt{\frac{D}{\left(\theta_{1}-\theta_{2}\right) \theta_{1} \theta_{2}}},
$$

что совпадает со значением числа вращения (19), полученным ранее.

Отметим, что для того чтобы все финитные орбиты имели одинаковое число вращения, необходимо, чтобы $C(\varepsilon)=C_{0}=$ const, т.е., по крайней мере, должно выполняться условие $C_{2}=0$. Это условие анализируется в следуюшем разделе.

\section{5. УРАВНЕНИЕ ДЛЯ ТРАЕКТОРИЙ НА СФЕРЕ. ЧИСЛЕННЫЙ АНАЛИЗ}

Следствием системы (7) является следующее уравнение второго порядка для угловой переменной $\Phi$, рассматриваемой как функция $\Psi$ :

$$
\Phi^{\prime \prime}=-\frac{1}{4 D} \operatorname{ctg} \Phi\left[\left(\theta_{2}-\theta_{1}\right)-\left(\zeta_{2}-\zeta_{1}\right) \Phi^{\prime}\right]\left(\zeta_{2} \Phi^{\prime}-\theta_{2}\right)\left(\zeta_{1} \Phi^{\prime}-\theta_{1}\right)
$$

Здесь $\Phi^{\prime \prime} \equiv d^{2} \Phi / d \Psi^{2}, \Phi^{\prime} \equiv d \Phi / d \Psi$. Из вида этого уравнения следует сушествование трех семейств траекторий на сфере, на которых $\Phi$ и $\Psi$ связаны линейной зависимостью. Рассмотрим на плоскости $(\Phi, \Psi)$ полосу $0 \leqslant \Phi \leqslant \pi$, являюшуюся разверткой рассматриваемой нами сферы. При этом верхняя и нижняя границы этой полосы соответствуют полюсам сферы, а средняя линия - ее экватору. Тогда указанным трем семействам траекторий отвечают на развертке сферы три семейства отрезков прямых, ограниченных верхней и нижней границами полосы и имеющих угловые коэффициенты

$$
k_{1}=\frac{\theta_{2}-\theta_{1}}{\zeta_{2}-\zeta_{1}}, \quad k_{2}=\frac{\theta_{2}}{\zeta_{2}}, \quad k_{3}=\frac{\theta_{1}}{\zeta_{1}} .
$$

Будем говорить о таких отрезках как об отрезках семейства 1, 2 или 3 , соответственно. Численный анализ показал следующее: если непрерывно изменять начальные условия так, чтобы полоса вокруг экватора, ограничивающая движение изображаюшей точки, расширялась, то гладкие финитные орбиты вырождаются в пределе в пилообразную ломаную, звеньями которой являются чередуюшиеся отрезки двух определенных 
семейств ( 1 и 2,1 и 3 или 2 и 3 , в зависимости от значений структурных параметров системы). Для краткости будем называть такие ломаные предельнымми цепочками. Таким образом, мы имеем три типа предельных цепочек: $(1,2),(1,3)$ и $(2,3)$.

В пространстве структурных параметров выделяются три области, связанные с определенным типом предельной цепочки. В одной из этих областей лежит, в частности, точка, отвечаюшая кеплеровскому случаю, в другой - точка, отвечаюшая гармоническому осциллятору, и в третьей - точка, отвечаюшая задаче Кеплера в импульсном представлении.

Прообраз предельной цепочки на сфере может оказаться замкнутым или незамкнутым в зависимости от значений структурных параметров. Отметим, что отрезкам, составляюшим цепочку, отвечают траектории на сфере, но не обязательно отвечают реальные траектории на исходной плоскости. Например, в кеплеровском случае предельные цепочки состоят из отрезка семейства 1 , отвечающего параболической траектории исходной задачи, и отрезка семейства 2 , не сопоставленного какой-либо траектории в исходной задаче.

Подчеркнем также, что два типа отрезков, составляюших одну предельную цепочку, отвечают траекториям на сфере, лежашим в разных уровнях интегралов.

Удобство введения предельных цепочек состоит в том, что им можно сопоставить число врашения $N_{\infty}$, равное удвоенной длине "зубца" цепочки, отнесенной к $2 \pi$, которое элементарно выписывается исходя из значений угловых коэффициентов $k_{m}$. Численный анализ подтверждает, что это число является пределом чисел врашения орбит финитных движений, вырождаюшихся (на развертке сферы) в предельную цепочку.

Для того чтобы все (финитные) орбиты имели одинаковые числа врашения, естественно потребовать, чтобы числа врашения, сопоставленные нами круговой орбите $\left(N_{0}\right)$ и предельной цепочке $\left(N_{\infty}\right)$, были равны. Как следует из результатов асимптотического анализа, другим необходимым условием является равенство нулю всех коэффициентов в разложении $C(\varepsilon)$, кроме $C_{0}$ (это условие не является, вообще говоря, априори независимым от первого). Численньй анализ приводит к следующему заключению: в классе гамильтонианов (3) совместное выполнение двух условий

$$
N_{0}=N_{\infty}, \quad C_{2}=0
$$

является необходимым и достаточным для того, чтобы орбиты всех финитных движений в системе имели одинаковые числа врашения. Если это число рационально, то все орбиты замкнуты.

Перечислим множества гамильтоновых систем класса (3), для которых выполняются условия (60).

В области, отвечающей цепочкам вида $(1,2)$, условия $(60)$ выполняются именно на двумерном многообразии (42) (см. п. 3.4). Приведем еше одну запись этого многообразия:

$$
\alpha_{1}-2 \alpha_{2}=2, \quad \beta_{1}-2 \beta_{2}=2 .
$$

Численный анализ подтверждает, что для любой системы с функцией Гамильтона (45) числа врашения всех орбит одинаковы и равны $2 /|D|$. 
В области, отвечающей цепочкам вида $(2,3)$, условия (60) выполняются на многообразии

$$
\alpha_{1}+\alpha_{2}=2, \quad \beta_{1}+\beta_{2}=2
$$

содержащем точку, отвечающую гармоническому осциллятору. Эта двумерная поверхность также расслаивается одномерными кривыми, вдоль которых сохраняется число врашения. А именно, число врашения равно $2 /|D|$ для всех орбит любой системы с функцией Гамильтона

$$
H=\frac{p^{1+D / 2}}{r^{-1+D / 2}}(r p)^{\gamma}+\sigma \frac{r^{1+D / 2}}{p^{-1+D / 2}}(r p)^{-\gamma}, \quad \sigma>0 .
$$

И, наконец, в третьей области пространства структурных параметров (с цепочками вида $(1,3))$ условия $(60)$ выполняются на двумерной поверхности

$$
\alpha_{2}-2 \alpha_{1}=2, \quad \beta_{2}-2 \beta_{1}=2
$$

Соответствующая функция Гамильтона получается из (45) заменой $r \leftrightarrow p$.

Численный анализ позволяет предположить, что указанными тремя двухпараметрическими семействами исчерпываются все системы из класса (3), в которых орбиты всех финитных движений в системе имеют одинаковые числа вращения.

Отметим, что, хотя построение таких многообразий в данной работе было основано на анализе неканонической динамической системы на сфере, нет сомнений в том, что эти же результаты могут быть получены путем анализа исходных канонических уравнений Гамильтона.

Благодарности. Авторы благодарят профессора В. И. Юдовича за полезные обсуждения результатов.

\section{Список литературы}

[1] Л. Д. Ландау, Е. М. Лифииц. Механика. М.: Наука, 1988.

[2] В. И. Арнольд. Математические методы классической механики. М.: Наука, 1989.

[3] V. M. Eleonsky, V. G. Korolev, N. E. Kulagin. The fractal oscillator and the Kepler problem. В сб.: Тезисы Международной конференции "Progress in Nonlinear Science" (Нижний Новгород, 2-6 июля 2001 г.). Ред. Л. М. Лерман, Л. П. Шильников. Нижний Новгород: Изд-во Нижегородского университета, 2002. С. 229-234.

[4] В. М. Елеонский, В. Г. Королев, Н. Е. Кулагин. О замкнутых орбитах и изохронных колебаниях в гамильтоновых системах вида $H=p^{\alpha_{1}} r^{\beta_{1}}+\sigma p^{\alpha_{2}} r^{\beta_{2}}$. В сб.: Тезисы Международной конференции "Нелинейные задачи теории гидродинамической устойчивости и турбулентность" (Москва, 10-17 февраля 2002 г.). Ред. С. Я. Герценштейн. М.: Изд-во Ин-та механики МГУ, 2002. C. $46-49$. 